\title{
How Information Shapes Portfolio Allocation During Financial Crises
}

\author{
Riccardo Boero \\ Los Alamos National Laboratory, \\ riccardo@lanl.gov \\ Marco Monti \\ IBM Italy, Milan - Vita-Salute San Raffaele University, Milan, \\ marco.monti@it.ibm.com
}

\begin{abstract}
During financial crises individual investors modify their portfolio allocation to decrease their exposure to more risky investments such as equities. Most recent empirical analyses of this phenomenon have focused on changes in risk attitudes, and in risk and return expectations but, so far, inconclusive evidence has been provided that changes in those psychological variables play a causal role in the actual decision making at work. Furthermore, the duration of the phenomenon, which is very short, is often not considered and it is thus unclear why the situation recovers so quickly after crises. Relying on experimental data and on a simple agentbased model, we propose an alternative explanation of this phenomenon based on the interaction of the two most important phases that individuals undertake in decision making, namely (1) searching information about possible options and (2) selecting the preferred one. Our main result is that the observed reallocation of portfolios in times of crisis is not the result of a portfolio reassessment driven by the crisis but it is instead explained by changes in search behavior for information on specific market conditions.
\end{abstract}

Keywords: Behavioral Finance, Investment Decision, Non-compensatory Heuristic, Financial Crisis, Experimentally-grounded Agent-Based Model 


\section{Introduction and Background}

Great financial crises that experienced between the mid of 2008 and the first quarter of 2009 worldwide are characterized by decreased returns for virtually all financial assets.

From a simplistic economic perspective, the dynamics is explained at the market level by a quick, deep and exogenous drop in equity markets, followed by downward dynamics in bonds and commodities due to lowered expectations about the real economy near future. According to this widespread approach, demand-side components play a relevant role through changes in expectations and "expected utility"-based decision making.

In recent years, the attention towards the role of investors and the availability of data collected during the 2008-2009 period has given raise to a multitude of empirical studies that lead to one important finding about individual investors' behavior, namely that during crises, portfolios held by individual investors are reallocated and that reallocation takes many forms. For instance, some investors in some countries prefer to concentrate their assets in their national financial markets while others prefer the opposite, that is to say to diversify their portfolio across foreign countries.

From the broader perspective of aggregated levels, two main stylized facts can be outlined. First there is a smaller percentage of allocation to equities and a reallocation towards other more liquid and less volatile assets ${ }^{1}$. Second the effect is very quick and short, as long as markets are going downward, and then it quickly recovers to the previous situation (Hoffmann et al. 2013a). Most interestingly, similar dynamics happen also for institutional investors that change their strategic, long-term (5-10 years) asset allocation (Pozen et al. 2011) towards fixed income and investments alternative to equities, perhaps suggesting a prompt and effective transmission of preference changes from their customers.

However, if we apply to those stylized facts the reasoning mentioned above for explaining the drop in all financial assets values, that is to say to explain them in terms of measures of expectations about financial markets and an utility driven decision making, the picture is rather confused.

For instance, Hoffmann et al. 2013b present evidence about the Dutch market that supports the idea that all important financial expectations are relevant, namely return expectations, risk tolerance and risk perception. Dorn \& Weber 2013 do not observe changes in risk perception in Germany during the period of interest. Weber et al. 2011, focusing on UK investors, support the risk-as-feelings hypothesis, according to which observed changes in risk taking behavior is associated with changes in risk and returns expectations but neither with recent experienced changes in stock market performance nor with risk tolerance. Merkle \& Weber

\footnotetext{
${ }^{1}$ Most recent testimonies of this empirical phenomenon can be found, for Germany, in Dorn \& Weber 2013 - see figure 3 for the period of financial crisis - and in Essner \& Rosenius 2012 for Sweden.
} 
2012, found that "investor beliefs have little predictive power for immediate trading behavior".

Similarly, if we consider the quick recovery after the crisis, the picture is not very much clearer. For instance, Hoffmann et al. 2013a critically discuss prior literature that asserts that crises can determine long lasting effects in risk taking due to long lasting changes in return and risk expectations, and in risk aversion, and in fact recovery can be quick because low asset prices represent good opportunities for other investors to enter the market (Hoffmann et al. 2013a , Dorn \& Weber 2013, Yu $\&$ Hsieh 2010). The problem is that we still do not know how expectations of new entrants have been affected by the crisis and why they should be different from the ones of market incumbents. Similarly, Calvet et al. 2009 focus on the importance of past experience and support the idea that risky assets are reallocated if have performed poorly, but in periods of crisis all financial investments present poor levels of expected returns and it remains unclear where households should reallocate their portfolios and, most importantly, when they should do that.

To say it differently, we believe that the literature on individual investments during times of crisis so far has largely failed in its main challenges, that is to say in clearly identifying the changes in expectations which are relevant and in mapping them to actual decision making capable to give raise to the two stylized facts mentioned above. So far the role of expectations in investment decision making during crises has not been identified, and having found evidence of loose correlations makes legitimate hypothesizing that such changes in expectations could be effects of reallocation, not its causes, or even just spurious correlations.

We thus propose to take a different perspective in order to explain the phenomenon. Our approach relies on acknowledging the role that information plays in decision making and it is consistent with the positive theory of simple and information-frugal decision rules proposed in Gigerenzer et al. 1999. Our work focuses on the hypothesis that information search is limited rather than exhaustive, and on how pieces of information are mapped into actual decisions. In particular, information-frugal decision making models classify and choose with only limited knowledge and computational effort, using subsets of available information. These models have been demonstrated to be effective for managing stocks portfolios (Borges et al. 1999) and for studying the behavior of non-professional investors (Monti et al. 2012).

In summary, with this work we aim at investigating whether limited information search and decision making based upon it explains the quick and short reallocation that characterizes individual investors' portfolios in times of crisis.

In particular we explore two specific research hypotheses about the role of information. First, we investigate the hypothesis that during financial crises investors "wake up" because of media and social pressure, and start monitoring and assessing the performance of their portfolios and they modify their allocation accordingly. In other words, we hypothesize that individual investors do not monitor their portfolios continuously, but they are urged to do so in times when 
they know that something "unusual" is happening in financial markets. Under the hypothesis, it is the unusual attention of investors on the performance of their investments that generates the observed change in portfolio allocation.

Second, we investigate another, completely different, hypothesis. In fact, we assume that investors continuously monitor their portfolios and we analyze whether it is the fact of receiving particularly negative information about returns currently obtainable in financial markets that actually changes portfolio allocation.

In the following section we introduce an experiment we conducted in a laboratory designed to test our first research hypothesis and to measure information search in different financial environments. Because experimental results refute the first research hypothesis, in the section that follows we present a simple agent-based model based on experimental data and aimed at investigating the second research hypothesis. The paper concludes summarizing results, discussing their limitations and their consequences from the broader perspective of financial advice and regulatory efforts. 


\section{An Experiment on Individuals' Investment Decisions}

Following increasing evidence that hypothetical decisions about investments have actual value (e.g. Monti et al. 2012 and Hoffmann et al. 2013b), we organized an experiment aimed at observing modifications in investing behavior induced by market feedbacks. Feedbacks, in particular, are received by experimental subjects in the form of the profits that chosen investments have obtained, as investors can experience in real life.

\subsection{Experimental Design}

Following the standards of experimental economics, the experiment used utility induced by monetary payoffs actually paid to subject in order to simulate a realistic financial environment. Furthermore, the experimental design was supported by a continuous interaction with a network of small Italian banks and with their customers in order to ensure high levels of realism and external validity.

The experiment was conducted in a sequence of three treatments. During the first one, subjects were asked to explore information and to take a decision about investments without receiving any feedback about the profits generated by their choices. In the second phase, such information was immediately sent back to subjects. The third treatment was equal to the first one (i.e., without any feedback) and aimed at measuring differences due to adaptations occurring in the second phase, i.e., by comparing observed behavior in the third and first phases. From now on, we call those three treatments "baseline", "feedback" and "final" phases for sake of simplicity.

The sequencing of the three phases is motivated by the research question we want to test experimentally, namely whether the availability of information about the performance of previous investment choices has an impact on portfolio allocation, under the assumption that in real world investors assess and modify their portfolios especially when financial crises happen. The comparison of the results obtained in the baseline treatment with the ones collected in the feedback and final phases in fact allows us to measure, respectively, how investments change during and after receiving information about investment performance.

Furthermore, it is important to stress how the adopted payoff scheme (taken from appendix A of Monti et al. 2012) allows modeling a financial environment ${ }^{2}$ that is realistic under our first research hypothesis. In fact, if we assume that during financial crises individuals assess the performance of portfolios they have selected over long periods in the past, there is no reason to believe that the distribution of investment returns, even if the evaluation happens in times of financial crisis, is anyhow skewed. To say it differently, it seems plausible to assume that returns resulting from such a reassessment are both high and low, and thus we adopted a

${ }^{2}$ For a more detailed description of the experimental design and a deeper discussion of its external validity please refer to Monti et al. 2012. 
probabilistic modeling of investment performance that distributes investment gains uniformly within the same class of assets.

In terms of options faced by the subjects, they were asked, in each treatment, to undertake three times a simple task of binary investment choice that is equal to the first one reported in Monti et al. 2012.

On each repetition of the task, two investments are randomly drawn from a set of six possibilities that represent the most common investments available to individual investors. The six investments are bank accounts (checking and savings), bank bonds and certificates of deposit, government bonds, insurance products with guaranteed capital, balanced mutual finds (with about 50-50 of blue-chip stocks and corporate bonds), and stocks. Each investment is characterized by six attributes: risk, time horizon, buying and management costs, liquidity, fixed income (coupons), costs for redemption, and exit fees. Each attribute would be assigned different values for each investment according to actual characteristics we identified with the help of the network of partner banks. For example, stocks are "high" risk while government bonds have a "low" risk value. The complete assignment of attributes to each investment instrument is presented in table 1.

\begin{tabular}{|r|c|c|c|c|c|c|}
\cline { 2 - 7 } \multicolumn{1}{c|}{} & Risk & $\begin{array}{c}\text { Time } \\
\text { Horizon }\end{array}$ & Costs & Liquidity & Coupon & $\begin{array}{c}\text { Redemption } \\
\text { Costs }\end{array}$ \\
\hline Bank Accounts & low & short & low & easy & yes & no \\
\hline Bank Bonds \& CDs & low & short & low & easy & yes & yes \\
\hline Government Bonds & low & medium & low & easy & yes & yes \\
\hline Insurance Products & low & medium & high & difficult & no & yes \\
\hline Balanced Funds & medium & long & medium & easy & no & no \\
\hline Stocks & high & long & medium & easy & no & no \\
\hline
\end{tabular}

Table 1. Possible investments and their characteristics.

Subjects did not know table 1 and did not know which investment options they were facing: in fact, their computer screen presented an empty $2 \mathrm{X} 6$ matrix where the two rows were labeled "investment A" and "investment B" and the columns headings reported all the six features, presented in a random order.

Subjects were asked to choose between investment pairs A and B and allowed to discover "one-by-one" the features of the two options in order to make an informed decision. Information exploration was expensive in terms of experimental currency units and thus by means of directly decreasing monetary payoffs actually earned by subjects in the experiment.

As mentioned above, in each treatment subjects faced the binary investment choice three times, in each of which two randomly chosen investments were available. The set of six possible investments and their features did not change across phases. The only difference between phases was the fact that during the feedback one subjects 
immediately received their payoff after each choice, thus receiving a feedback about the outcome of each of their choices.

The subject pool comprised 56 students (61\% maled) enrolled in an undergraduate program in Business Administration at the University of Torino, Cuneo campus. Subjects were not allowed to communicate and they interacted only with their personal computer in order to access the experiment implemented in zTree (Fischbacher 2007). The experiment lasted about 15 minutes and on average subjects earned about 10 euro each ${ }^{3}$.

\subsection{Experiment Results}

The first outcome of the experiment is that, on average, treatments have only a very small impact. In fact, comparing average payoffs received across phases does not reveal large differences ${ }^{4}$ (baseline vs. final: $p=0.4021$; baseline vs. feedback: $p=$ 0.3032 ; feedback vs. final: $p=0.7920$ ). The distribution of choices, in terms of the portfolio derived from subjects' choices, does not show differences either. For instance, for the case of allocation to stocks, their average percentage in the aggregated portfolio does not decrease after the feedback phase (baseline: $11.1 \%$, feedback: $10.5 \%$, final: $13.0 \%$; $p$ values referred to differences between treatments: baseline vs. final 0.8527 , baseline vs. feedback 0.6121 , feedback vs. final 0.4927 ).

Experimental results thus allow us to refute the first hypothesis we have identified about the role of information in individuals' asset allocation in times of financial crisis. In fact, observed changes in the aggregated portfolio are not explainable as resulting from a reallocation of portfolios of individuals who are called by recent market developments to re-assess and update their investments.

Nevertheless, the only relevant impact of the feedback phase is on information search. In fact, if we compare the average percentage of information exploration, in terms of the number of attributes subjects searched over the twelve possible in each investment task, we note that it is 34.6 in the baseline treatment and 31.7 in the final treatment $(\mathrm{p}=0.0541)$.

Moreover, computing the percentage of information exploration for each investment feature, we find small but important decreases for time horizon, liquidity and redemption costs as reported in table 2 .

${ }^{3}$ Experimental payoffs have been computed as described in appendix A of Monti et al. 2012.

${ }^{4}$ The tests from now on presented on experimental data have been computed using the Wilcoxon matched-pairs signed-ranks test as pairs are matched due to the "within" design of the experiment. 


\begin{tabular}{|r|c|c|c|}
\cline { 2 - 4 } \multicolumn{1}{c|}{} & Baseline Phase & Final Phase & p Values \\
\hline Risk & 65.8 & 70.5 & 0.1781 \\
\hline Time Horizon & 27.7 & 18.1 & 0.0064 \\
\hline Costs & 24.7 & 30.6 & 0.1856 \\
\hline Liquidity & 31.5 & 21.7 & 0.0106 \\
\hline Coupon & 44.6 & 41.7 & 0.5974 \\
\hline Redemption Costs & 12.8 & 7.1 & 0.0368 \\
\hline
\end{tabular}

Table 2. Percentage of explored information over investment features in the baseline and final phases ( $p$ values refer to the difference between values in the two phases).

Similarly, if we look at the Overlapping Information Index (OII - Monti et al. 2012) that measures the percentage of identical features looked up for both investment options, we can notice on average a slight increase ( $47.7 \%$ in baseline, $53.2 \%$ in final $-\mathrm{p}=0.0992$ ).

Overall, we can conclude that the fact of receiving feedbacks about market returns per se does not significantly affect investments allocation (and performance) in the short run, and that there is only a small impact on information search without further consequences.

We thus answer negative the first research question. However, there is a second hypothesis to investigate. In fact, abandoning the previous hypothesis we can assume that investors frequently assess and update their portfolios and we can investigate whether market conditions affect information searched for and the resulting portfolio allocation.

In order to address that second research hypothesis we need to differentiate between the kind of feedbacks investors' receive under different market conditions. In fact, in the experiment we modeled feedbacks about investment performance as uniformly distributed because we assumed that investors were evaluating the performance of assets cumulated over their investment life. However, if investors continuously evaluate the performance of different assets over time, it is plausible to assume that they focus on investment performance as it is obtainable in contemporaneous market conditions. Because we are focusing on times of crisis, we need to look at market conditions where assets returns are particularly skewed.

We identify two archetypal market conditions. In the first one, from now on called the "bear" condition, mostly negative feedbacks are received, that is to say that all investments perform poorly, earning low yields relative to the average value of their long term distribution. The opposite situation is the "bull" condition, that is to say when the large majority of investments earn high returns.

In order to study those two conditions, we select appropriate clusters of subjects in our experimental sample. In particular, for each investment choice by subjects during the feedback phase we compute the difference between its expected yield 
(i.e., the average value of the return distribution) and the actual payoff, which is uniquely due to random variables automatically drawn by the program managing the experiment. The difference between actual and average yields is a measure of how "lucky" the subject has been, or of the exogenous positive or negative trends in financial markets that temporarily shift yields to the tails of their long term distribution.

We then sum up such values over the three repetitions of investment choices each subject undertook during the feedback phase and we select the two groups of individuals in the percentiles between $0 \%$ and $10 \%$ and between $90 \%$ and $100 \%$ of the distribution of this latter variable. The former group is the one made by subjects in the bear condition and the latter is the subset of subjects in the bull condition.

It is interesting to observe that portfolios allocated by subjects during the feedback phase do not differ in the two market conditions, confirming that the difference in performance is only a matter of chance and not of investing behavior. Moreover, the subset of our subjects we do not consider further (i.e., subjects neither included in the bear nor in the bull market condition) has faced an environment where yields are balanced, sometimes higher and sometimes lower than average, without a clear market trend. On the contrary, subjects in the bear condition have faced an environment in which all assets yield low returns, if not negative ones, and subjects in the bull condition have faced only good returns, relatively to the ones that can be obtained in our experimental design.

We mentioned that portfolios of subjects in the two conditions during the feedback phase do not differ5: the opposite happens if we compare the baseline and the final phase for individuals in the bear condition. In particular, stocks $(p=0.0833)$, that is to say the risky investment option, see their weight in those subjects' portfolios decline. Such a difference looks very like the first stylized fact described above about financial crises, and it could be due to different information search, different investment selection, differences in both or, lastly, to differences in unobserved variables such as expectations.

Looking at experimental data about information search, the percentage of explored cues during the final phase differs between the two market conditions $(p=0.0022)$. In particular individuals in the bull condition explore just $20.6 \%$ of available information while individuals in the bear condition explore more (37.8\%). Similarly, there is a difference in the OII index ( $p=0.0005)$ : it is $72.8 \%$ in the bear condition and just $20.6 \%$ in the bull one.

In summary, in the two conditions information search is different as subjects who have faced a hard, negative, financial environment search for much more information and they try to overlap it in order to compare investment options on the same features.

${ }^{5}$ Statistical tests on the two market conditions and on the results obtained from the agent-based model presented below have been conducted using the two-sample Wilcoxon rank-sum (Mann-Whitney) test as individuals (and agents in the model) exclusively belong either to the bull market condition or to the bear one. 
Is the change in information search therefore responsible for different allocations when there are different trends in financial markets? Although the evidence we collected in the experiment seems to support that hypothesis, we need something more to support that changes in information search are sufficient to explain the reallocation of individual investors portfolios in times of crisis and that variables unobserved in our experiment (e.g. expectations) are not essential to explain the phenomenon. 


\section{The Experimentally-grounded Agent-based Simulation}

In order to test whether changes in information search in times of crisis can generate at the aggregated level the reallocation of portfolios, we prepared a simple agent-based model.

We claim that our model is very simple from the viewpoint of the agent-based approach because of two reasons. First, it does not have any kind of interaction among agents, as they only interact with the financial environment. Second, there is not any path-dependence as agents have no memory and they are not capable of changing the environment with which they interact.

The model, furthermore, is experimentally-grounded as it uses data collected during the experiment in order to model information search behavior in agents, as explained in details in the following paragraph.

\subsection{Model Structure}

The model ${ }^{6}$ can be described in terms of its two main elements, agents and their environment. Starting from the latter, it is defined by six investments and their features as described in table 1. Furthermore, a random function selects a couple of investments among the six available ones on every simulation step and for each agent. Obviously, investment features are hidden from agents' eyes, and it is a decision of agents whether to search for information. The investment environment faced by artificial agents is thus exactly similar to the one presented to human subjects in the experiment.

Agents are defined by two behavioral components. They do not have any kind of heterogeneity in their endowment because they represent individual investors asked to choose one investment of the same value on each simulation step and because our focus is on comparative portfolio allocation and not on variations of the total value of assets.

The two components of agents (investors) behavior are information search and investment choice.

We model information search behavior of agents in the form of discrete-time Markov chains calibrated over experimental data. In information search, and in the Markov chain, agents can be in 7 possible states. Six states correspond to the six features that characterize every investment. In the last state (the seventh), information search stops. Furthermore, there is a starting state in which agents do not know anything about the two possible investments. After that initial state, they pass from state to state according to a probabilistic behavior until they reach the last state. Obviously, the fact of being in a state during the Markov chain means, for that agent, having searched for the corresponding investment feature.

${ }^{6}$ The agent-based model has been developed using Netlogo (Wilensky 1999). 
The behavioral loop of information search has a further component to effectively implement the Markov chain: whenever a state corresponds to an investment feature, and not to the stopping state, three outcomes can happen. First, if that feature is unknown for both investment options, one option is randomly chosen and the corresponding feature value is revealed to the agent. Second, the new state can correspond to a feature the value of which is known for one of the two investment options (that is to say that the agent has already been in that state during the Markov chain), and in that case the agent is provided with the feature value of the other, unknown, investment option. Lastly, if both values of that feature are already known, the agent ignores the selection of that state and probabilistically selects a new state.

Finally, when the Markov chain ends in the stopping state, information search is concluded and the agent is asked to make a decision based on the information discovered during the path from state-to-state of the Markov chain.

Markov chains implemented in the model for information search use transition matrixes observed during the experiment for subjects in the two market conditions, as reported in table 3 .

Bear Market Condition

\begin{tabular}{|r|c|c|c|c|c|c|c|}
\hline & Risk & $\begin{array}{c}\text { Time } \\
\text { Horizon }\end{array}$ & Liquidity & Coupon & Costs & $\begin{array}{c}\text { Redemption } \\
\text { Costs }\end{array}$ & Stop \\
\hline Start & 0.67 & 0.20 & 0.00 & 0.07 & 0.07 & 0.00 & 0.00 \\
\hline Risk & 0.48 & 0.07 & 0.07 & 0.19 & 0.11 & 0.00 & 0.07 \\
\hline Time Horizon & 0.29 & 0.29 & 0.14 & 0.14 & 0.14 & 0.00 & 0.00 \\
\hline Liquidity & 0.00 & 0.00 & 0.29 & 0.00 & 0.29 & 0.00 & 0.43 \\
\hline Coupon & 0.06 & 0.00 & 0.13 & 0.44 & 0.06 & 0.00 & 0.31 \\
\hline Costs & 0.09 & 0.00 & 0.00 & 0.18 & 0.27 & 0.00 & 0.45 \\
\hline Redemption & 0.00 & 0.00 & 0.00 & 0.00 & 0.00 & 0.00 & 1.00 \\
\hline
\end{tabular}

\begin{tabular}{|r|c|c|c|c|c|c|c|}
\hline & Bull Market Condition \\
\hline Rtart & 0.47 & 0.00 & 0.07 & 0.13 & 0.20 & 0.00 & 0.13 \\
\hline Risk & 0.07 & 0.07 & 0.27 & 0.20 & 0.07 & 0.00 & 0.33 \\
\hline Time Horizon & 0.50 & 0.00 & 0.00 & 0.00 & 0.00 & 0.00 & 0.50 \\
\hline Liquidity & 0.20 & 0.20 & 0.00 & 0.00 & 0.00 & 0.00 & 0.60 \\
\hline Coupon & 0.13 & 0.25 & 0.00 & 0.25 & 0.00 & 0.00 & 0.38 \\
\hline Costs & 0.00 & 0.00 & 0.00 & 0.00 & 0.00 & 0.00 & 1.00 \\
\hline Redemption \\
Costs
\end{tabular}

Table 3. Transition matrixes used by Markov chains representing investors' information search. 
Markov transition matrixes as the ones presented above are easy to be understood and have to be read row by row. The state in which an agent is appears on the first column (i.e., the column containing rows labels). Then, the probability of moving from that state to others is represented by values presented in the same row. For instance, individuals in the bull market condition at the beginning (i.e., at the starting state, the row labeled "start") have the largest probability (47\%) to search for information about the risk of investment options. Obviously, the sum of probabilities over each row equals 1 in order to determine with certainty the following state in the Markov chain. Transition matrixes presented in table 3 are the ones used in the model and they derive from observed distributions of information search choices made by experimental subjects belonging to the two market conditions.

The behavior just described allows agents to partially discover information about investment options presented by the environment. Agents then have to select one of the two options and they do that according to a fast and frugal heuristic that has been introduced in Monti et al. 2012 that guarantees accuracy in predictions of decisions for individual investors engaged in binary investment decisions.

Synthetically, the heuristic is a hybrid between lexicographic and tallying rules. In particular, the lexicographic part is predominant and considers only information about the level of risk of investment options. The tallying component of the heuristic is used only if the lexicographic part does not lead to a decision and it compares the options by summing up, with equal weights, the positive features of investments choosing the one with the highest value. In order to apply the tallying rule, value of investment features are transformed in 0 s and $1 \mathrm{~s}$, where 1 indicates a positive value according to preferences we observed in interviews with individual investors. For instance 7 , if time horizon is "medium" or "short" its new value is 1 , while it is equal to 0 if it is "long" as investors consider, ceteris paribus, longer time horizon a negative feature.

The behavioral model for binary investment selection, which is represented in figure 1 and that has been validated on samples of household investors holding at least 40,000-euro portfolios and university students without prior experience in portfolio management (Monti et al. 2012), predicts that for individual investors there is no compensating trade-off for high risk investments if alternatives with lower risks are considered.

\footnotetext{
${ }^{7}$ For a more detailed discussion of the heuristic, of the transformation of investment features needed to use it and of its validation refer to Monti et al. 2012.
} 


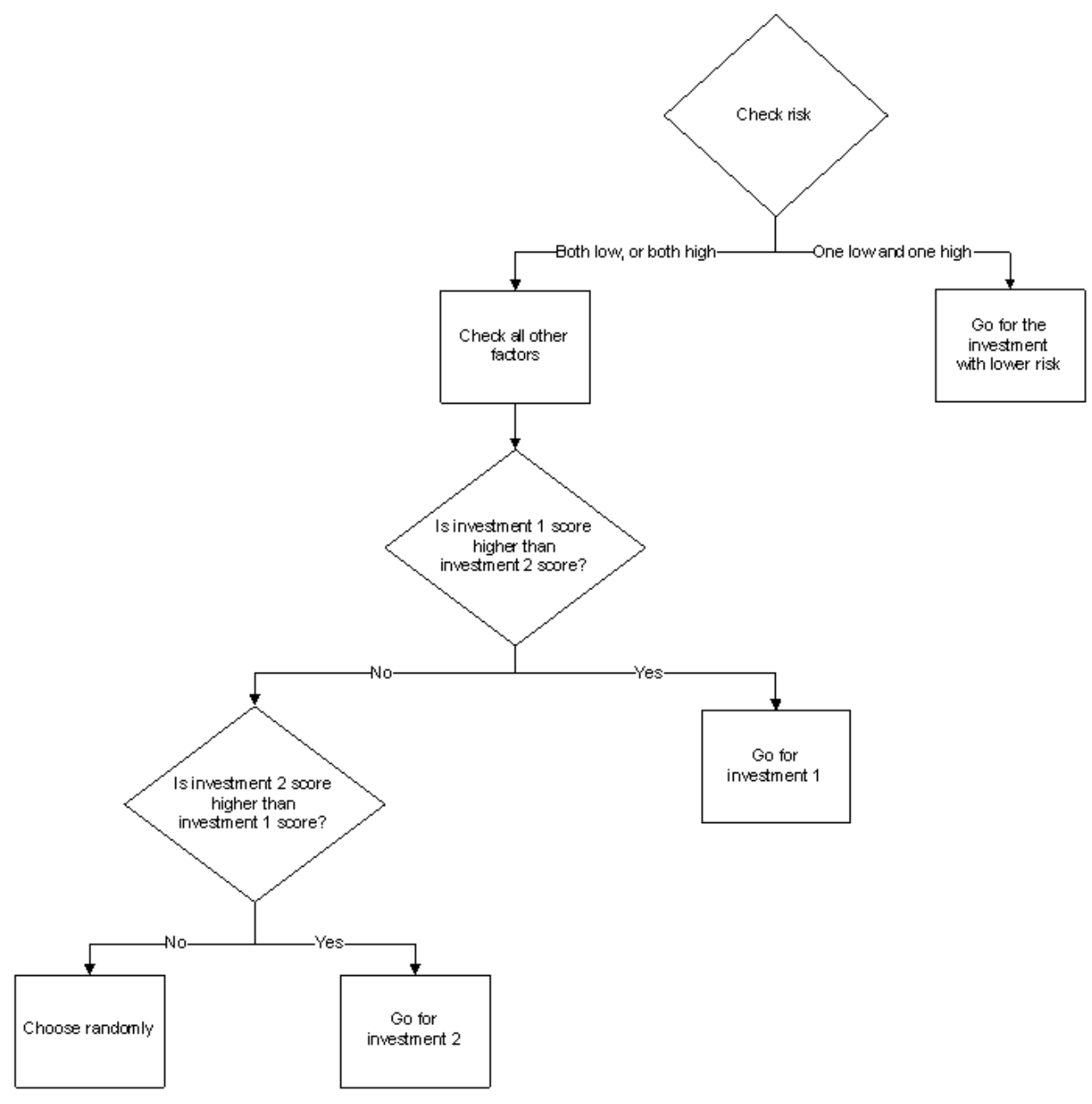

Figure 1. Fast and frugal tree for binary investment decisions (from Monti at al. 2012).

In summary, model agents face the same financial environment used in the experiment and that has been designed to be as close as possible to actual household investors' choices. They can search for information about the options they have at disposal and then they choose an investment.

Information search is modeled as a Markov chain calibrated on data observed in the experiment for the two market conditions, bear and bull. Investment selection is modeled with a fast and frugal heuristic that effectively models binary investment decisions. The model can be run with different values for its parameters. In particular, it can be run with bull or bear market conditions, with a different random seed for the random number generator that is used in probabilistic algorithms and with a different number of investors. 


\subsection{Simulation Results}

We conducted the analysis by comparing results obtained in the two market conditions, with 1,000 agents, over 100 simulation time steps and with 10 different random seeds. It is worth noting that we analyze results obtained with these parameters as they guarantee enough data to neutralize the determinism that could be introduced by the artificial random numbers generator. In fact, with parameters values we have used we can compare data from 1,000,000 choices in each market condition.

Results are presented in figure 2: in each asset class there are differences between the two market conditions with $\mathrm{p}=0.0000$.

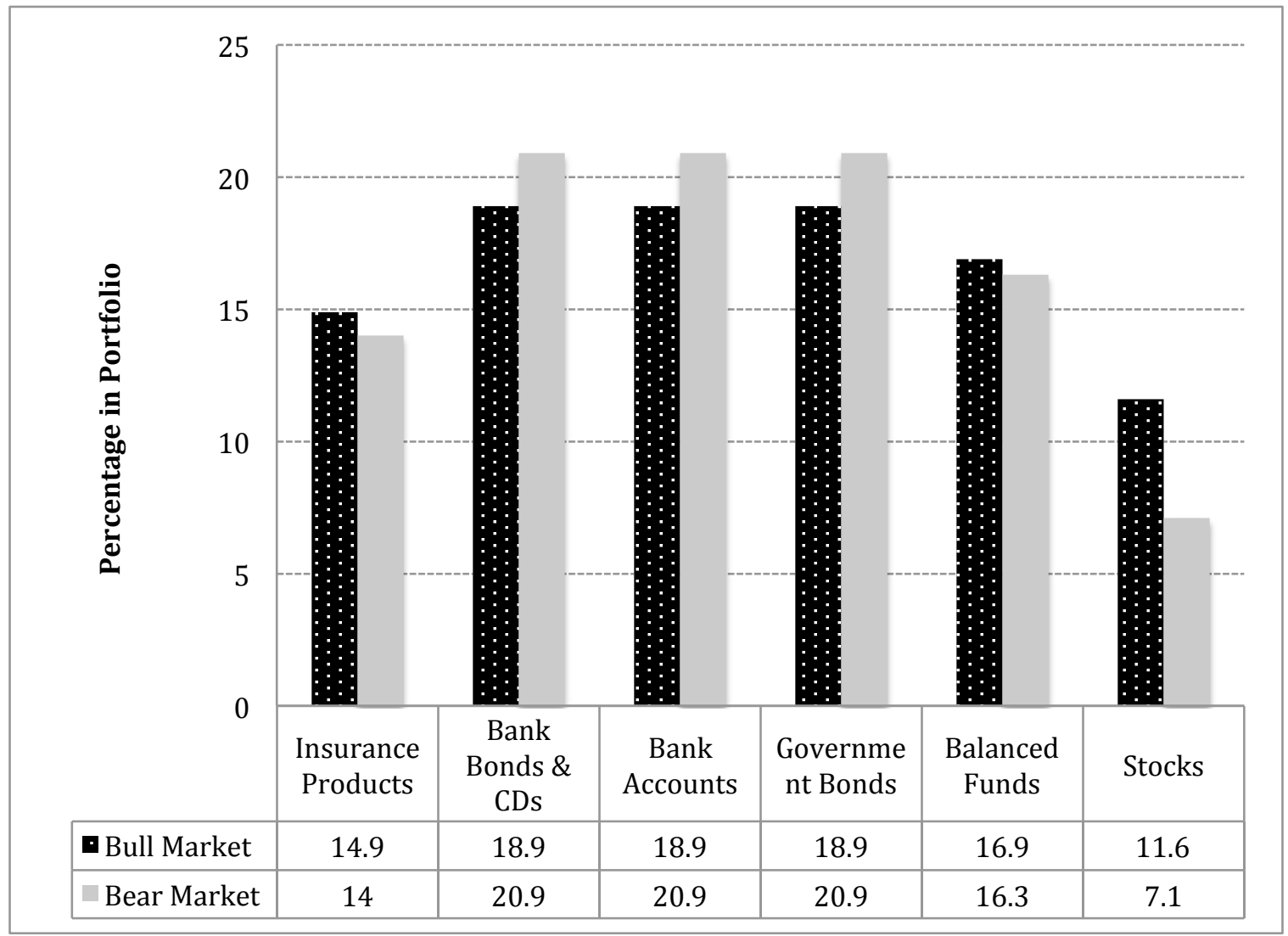

Figure 2. Average distribution of simulated portfolio allocations over investment type, in bull and bear market conditions.

As results presented in the figure clearly show, a bear market condition generates one of the stylized facts of actual portfolio allocation by individual investors in times of crisis, namely, substituting equities for other kinds of investments (i.e., fixed income and more liquid assets).

Model results, furthermore, are consistent with the other stylized fact of financial markets we pointed out at the beginning, namely, that individual investors' 
reallocation happens quickly. In fact, as soon as financial markets turn bear, investors move away from equities. The opposite occurs as soon as markets recover, e.g., exogenous shocks such as new investors entering financial markets attracted by low asset prices lead investors to move to equities.

Overall, the model allows us to conclude that the small behavioral change we observed in the experiment is sufficient to explain the reallocation of assets during crises since we do not need to assume changes in other parts of investors' behavior (e.g., investment selection or long term return expectations) to replicate the phenomenon. Model results strengthen what we observed in experimental data because during the experiment we could not control for subjects' behavior in investment selection. 


\section{Discussion}

In this work we have shown that individual investors' reallocation of investment portfolios during financial crises can be effectively explained in terms of changes in the information searched for and considered to make a choice. Our results suggest that often, in order to understand a phenomenon resulting from dispersed decision making, a research strategy directly aimed at investigating the actual decision making at work is crucial.

In particular we first have shown that the hypothesis that investors reassess and modify their portfolios rarely except during financial crises is not consistent with observed stylized facts about aggregated portfolio allocation. Second, we have shown that, on the contrary, investors continuously keeping track of the performance of their portfolios modify their choices when financial markets are in crisis because of the information they receive on the poor yields obtainable by all asset classes. This mechanism, furthermore, does not require changes in behavior regarding investments selection as changes in information search are sufficient to explain the phenomenon.

Finally, we have shown that approaches that are plausible both from the viewpoint of the frugality of information processing and from the one of the cognitive effort required for decision making can be enriched, from an analytical perspective, from the conjoint use of field and experimental data and of formal models such as agentbased ones which allow to verify hypotheses derived from collected evidence.

From the point of view of behavioral finance, the fact of having shown that changes in information search behavior in investors are sufficient to generate realistic macro outcomes reinforces previous analyses (e.g. Borges et al. 1999 and Agnew \& Szykman 2005) pointing out that information availability has a leading role in shaping investors' decisions. Similarly, our work enriches analyses of the role of media during financial crises (e.g. Casarin \& Squazzoni 2013).

Our result also has very important consequences for policy design and for business organizations, in particular in the field of financial advising. Since individual investors are so influenced by the information they receive about assets returns and since that can impact their risk exposure so deeply, it is of uttermost importance that regulatory agencies, banks and other institutions providing financial advice explicitly acknowledge and take into consideration the role of information. For instance, in the MiFID directive of 2004 (Markets in Financial Instruments Directive, regulating investment services in 30 European countries) such awareness was largely still lacking, leaving space for strategic manipulations of customers' risk exposure by financial advisers.

Our analysis, however, presents several limitations. First, in both our experiment and model we do not take into consideration possible feedbacks from market changes induced by individual behavior. In fact, we used assets returns as triggers for changes in individuals' behavior, but we do not know if changes in individual behavior provoke changes in markets beyond aggregated allocations. In other terms, we observe an emergence at the macro level by means of micro decision 
making and we also have an influence on micro decision making by the macro level (Gilbert 1995). However they are not connected because macro variables are different (i.e., returns and aggregated portfolio allocation) and the top-down influence is completely exogenous (and a model parameter).

Second, we did not consider in depth the presence of heterogeneity in investors. The reason for our approach is our focus on ecologically rational decision making (Gigerenzer \& Selten 2002). However, it may be interesting to evaluate the presence of heterogeneity in investors' behavior and performance, in particular if the research effort is addressed at dynamics such as entering the investment market, modifying personal financial attitudes (O'Neill and Xiao 2012), adapting investment behavior (Seru et al. 2010), or on the heterogeneity in risk exposure.

In conclusion, our results are meaningful in the context of relatively short financial crises such as the one in the 2008-2009 period. If, for any reason, new uncertainties and instabilities will affect financial markets and assets returns for longer periods of time, our results can become less significant because experienced lower returns have been observed to influence investing behavior over the long run (Malmendier \& Nagel 2011).

\section{Acknowledgments}

We thank Davide Donati, general director of the Cassa Rurale Giudicarie Valsabbia Paganella, and all the Board of Executives, for their full support in conducting onsite research at their banks. We acknowledge financial support from Regione Piemonte [IIINBEMA Research Project]. 


\section{References}

Agnew J.R., and Szykman L.R. (2005), “Asset Allocation and Information Overload: The Influence of Information Display, Asset Choice, and Investor Experience”, Journal of Behavioral Finance, 6(2): 57-70.

Borges B., Goldstein D.G., Ortmann A., and Gigerenzer G. (1999), “Can Ignorance Beat the Stock Market?”, in G. Gigerenzer, P.M. Todd, and the ABC Research Group, 'Simple Heuristics That Make Us Smart', Oxford University Press.

Calvet L.E., Campbell J.Y., and Sodini P. (2009), "Fight or Flight? Portfolio Rebalancing by Individual Investors", The Quarterly Journal of Economics, 124(1): 301-348.

Casarin R. and Squazzoni F. (2013), "Being on the Field When the Game Is Still Under Way. The Financial Press and Stock Markets in Times of Crisis". PLoS ONE, 8(7): e67721.

Dorn D., and Weber M. (2013), “Individual Investors' Trading in Times of Crisis: Going It Alone or Giving Up?”, Working Paper.

Essner N., and Rosenius N. (2012), "The Financial Crisis effects on asset allocation A study regarding the individuals in Umea financial behaviour in response to the financial crisis of 2008", Bachelor Thesis, Umeå School of Business and Economics.

Fischbacher U. (2007), “z-Tree: zurich toolbox for ready-made economic experiments", Experimental Economics, 10(2): 171-178.

Gigerenzer G., Todd P.M., and the ABC Research Group (1999), 'Simple Heuristics That Make Us Smart', Oxford University Press.

Gigerenzer G., and Selten R. (2002), 'Bounded Rationality: The Adaptive Toolbox', MIT Press.

Gilbert N. (1995), “Emergence in Social Simulation” in Gilbert N. and Conte R. (eds.) 'Artificial Societies: The Computer Simulation of Social Life, UCL Press, London, 144156.

Hoffmann A.O.I., Post T., and Pennings J.M.E. (2013a), "Individual investor perceptions and behavior during the financial crisis", Journal of Banking \& Finance, 37(1): 60-74.

Hoffmann A.O.I., Post T., and Pennings J.M.E. (2013b), “How Investor Perceptions Drive Actual Trading and Risk-Taking Behavior", Journal of Behavioral Finance, forthcoming.

Malmendier U., and Nagel S. (2011), "Depression Babies: Do Macroeconomic Experiences Affect Risk Taking?", The Quarterly Journal of Economics, 126(1): 373416.

Merkle C. and Weber M. (2012), "Do Investors Put Their Money Where Their Mouth Is? Stock Market Expectations and Investing Behavior", (August 22, 2012). Available at SSRN: http://dx.doi.org/10.2139/ssrn.1786456 
Monti M., Boero R., Berg N, Gigerenzer G., and Martignon L. (2012), “How do common investors behave? Information search and portfolio choice among bank customers and university students", Mind \& Society, 11(2).

O’Neill B., and Xiao J.J. (2012), "Financial Behaviors Before and After the Financial Crisis: Evidence from an Online Survey", Journal of Financial Counseling and Planning, 23(1): 33-46.

Pozen R.C., Palmer B., and Shapiro N. (2011), "Asset Allocation by Institutional Investors after the Recent Financial Crisis", in Y. Fuchita, R. Herring, and R. Litan (eds.) 'Growing Old: Paying for Retirement and Institutional Money Management after the Financial Crisis', Brookings Institution Press.

Seru A., Shumway T., and Stoffman N. (2010), "Learning by Trading", Review of Financial Studies, 23(2): 705-739.

Weber M., Weber E.U,. and Nosic A. (2013), "Who Takes Risks When and Why: Determinants of Changes in Investor Risk Taking", Review of Finance, forthcoming.

Wilensky U. (1999), NetLogo, http://ccl.northwestern.edu/netlogo/. Center for Connected Learning and Computer-Based Modeling, Northwestern University. Evanston, IL.

Yu H., and Hsieh S. (2010), "The effect of attention on buying behavior during a financial crisis: Evidence from the Taiwan stock exchange", International Review of Financial Analysis, 19(4): 270-280. 